\title{
Der Trinker
}

\section{von Monte Casso}

\section{Erzählung aus der Sammlung "Vom Satteln der Nilpferde»}

Teil 2

\section{A. J. Koemeda}

$\mathrm{V}$

Die Zaunreparatur gestaltete sich etwas schwieriger als ich dachte.

- Werner, sei nicht so pedantisch, sagte ich zu ihm nach etwa zwei Wochen. - Es handelt sich doch nur um die Stellen, an die der neue Zaun kommt. Alles aufräumen, eine tolle Ordnung im ganzen Garten haben, okay, das kannst du schon machen ... warte aber ab, bis wir fertig sind.

Wenn er sich etwas in den Kopf gesetzt hatte, liess er nicht mit sich reden.

- Kommt nicht in Frage! sagte er. - Wenn der alte Zaun mit dem Efeu und den Büschen weg ist - dann ist auch die Sichtsperre weg, dann muss hier Ordnung sein. Überall! Die Leute sollen nicht meinen, dass ich in einem Saustall lebe.

Ich bot ihm meine Hilfe beim Aufräumen an, er lehnte aber ab.

- Du kannst dabei nichts machen, Helmut, du weisst doch gar nicht, was wohin gehört. Nein, lieber nicht, du würdest mir nur im Wege stehen!

Bald danach holte er bei mir das italienische Geld für das Material - es war ein kleinerer Betrag, als ich gedacht hatte. Vielleicht hatte er beim Gärtner Secondhand-Ware gefunden ... nein, ich fragte ihn nicht danach.

Wir wollten eine Zwischenlagerung bei mir vornehmen und dann - bei schönem Wetter - mit dem Transport zur "Villa» anfangen. Dass ich einen Schubkarren benützen würde, war Werner auf einmal nicht so wichtig; er meinte nur, dass er selber kein derartiges Instrument anfasse, ja, davon dürfe ich ruhig ausgehen. Das müsse mich aber nicht stören ... er werde auf alle Fälle das meiste alleine hintragen.

- Gut, Werner, ich bin einverstanden.

Und der bunte Plastiksack?

Den hatten wir vergessen! Beide! Erst als ich zwei Tage später mit meinem Schubkarren zu Werners Villa fuhr, sah ich plötzlich das Ding an einem Ast im Wald hängen; ein wenig versteckt und trotzdem - von unserem Transportpfad aus gerade noch sichtbar.

Korrespondenz:

Dr. med. Adolf Jens Koemeda

"Breitenstein»

CH-8272 Ermatingen
- Werner, was meinst du, wie lange soll der Sprengstoff da baumeln?

- Häh? Was sagst du?

- Der Sprengstoff, Semtex vielleicht. Ich weiss nicht, was du alles darin vermutest ... non capisci? Ich spreche doch von dem Plastiksack. Siehst du ihn nicht? Da baumelt er. Du hast ihn selber dort hingehängt!

Werner blieb stehen und warf die Pfähle auf den Boden.

Alles vergessen! Er wirkte ratlos.

- Was meinst du, Helmut?

- Ich glaube, wir sollten nicht übertreiben. Wo siehst du noch ein Problem? Der Sack hing doch die ganze Zeit draussen, im Regen. Zwei oder drei Wochen lang. - Ja ...

- In dem Fall besteht für uns bestimmt keine Gefahr mehr. Alles ist nass ... der Sprengstoff, falls es sich tatsächlich darum handelt, ist total nass!

- Meinst du?

- Das meine ich, Werner, das meine ich wirklich!

- Und jetzt? Was machen wir?

- Lass uns überlegen!

Eine ausführliche Beratung - nach einer milden Stärkung - war natürlich angesagt. Wir bedachten die verschiedensten Aspekte der Ausführung und die versteckten Gefahrenquellen; zuletzt entschieden wir uns für das Mutige: Man werde den Sack vom Baum herunternehmen, vorsichtig öffnen und den Inhalt gründlich untersuchen. Jawohl!

Werner wirkte wieder aufgeregt; er wollte zwar auch diesmal selber anpacken, seine Angst war aber zu gross. Er überliess mir, immer noch zögernd, die Hauptrolle.

Mit einem langen Stock nahm ich den Sack herunter und legte ihn auf den moosigen Boden.

- Vorsichtig, Helmut! Langsam und sehr vorsichtig! Ich breitete den oberen Teil des Plastiksacks aus und schaute hinein. Ein Brief. Und darunter: Ein Paket. Etwa zehn mal zwanzig Zentimeter gross, fest zusammengerollt, drei oder vier Gummiringe hielten das wurstartige Gebilde zusammen.

- Also? Was siehst du?

- Einen Briefumschlag. Und ... darunter ein längliches Paket.

- Das lass noch drin! Den Brief allerdings ...

- Keine Angst, Werner, jetzt kann nichts schiefgehen. Alles ist ... nun, nicht direkt nass, aber ziemlich feucht.

Langsam nahm ich den Briefumschlag heraus. Federleicht, keine Hinweise auf einen problematischen Inhalt.

- Du, das fühlt sich gut an! Dünn und weich, darin kann höchstens ein Blatt sein ... willst du ihn nicht selber öffnen?

- Du hast angefangen! Mach weiter!

Mit meinem Taschenmesser öffnete ich die längliche Seite des Umschlags. Darin ein hellrosa Blatt; ich zog es heraus und entfaltete es.

- Italienisch!

- Also lies! Du kannst es gleich übersetzen. 
E per te, mio bravo poverino. Übersetzt etwa: Es ist für dich, mein guter Poverino ... Poverino verstehst du doch selber, Werner!

Er streckte die Hand aus, ich reichte ihm das rosa Papier. Er las es. Zuerst halblaut, dann nur mit den Augen.

- Für mich also?

- Für wen denn sonst? Das Ganze hing an deiner Tür!

- Mach weiter!

- Willst $d u$ nicht weitermachen, Werner? Wo liegt das Problem? Darin ist vermutlich ein Geschenk.

- Mach nur du! Schnell, bitte!

Ich griff in den Sack und zog das Paket heraus.

- Nicht sehr schwer. Sprengstoff müsste ein ganz anderes Gewicht haben!

Werner winkte es herbei. Eine kurze, ungeduldige Bewegung mit der rechten Hand. Ich gab ihm das hellbraun eingepackte Ding. Er schaute es sich von allen Seiten an, roch daran, dann legte er es an sein linkes Ohr und lauschte.

- Kein Ticken, nichts? fragte ich.

Keine Antwort.

Er streifte die drei roten Gummibänder ab, nahm das bräunliche Packpapier weg und legte es zwischen uns auf den Boden. Endlich - sehr langsam und vorsichtig - entfernte er die nächste, diesmal weisse, Papierschicht. Eine hellbeige Schachtel, etwa zehn mal drei Zentimeter gross, kam zum Vorschein.

- Also weiter, Werner!

Er tat nichts. Erst nach einer Weile: - Lieber du, Helmut!

- Nein, nein! Jetzt bist du schon ziemlich weit gekommen. Den letzten Schritt kannst du auch machen!

Er wollte nicht. Das Ganze sehe schon ein wenig verdächtig aus, flüsterte er, das müsse ich doch selber zugeben.

- Ach Quatsch! Nicht die Spur! Gib dir jetzt einen Ruck. Weiter um Gottes Willen!

Er zögerte immer noch. Sein Vorschlag: Die Schachtel unter dem Baum liegen zu lassen und aus einer gewissen Entfernung - sechs, sieben Meter müssten reichen - einen Stein auf das Ding zu werfen. Wenn nichts passiere, keine Explosion ... gut, dann könne man weiter schauen.

- Unsinn, Werner, totaler Unsinn! Dort könnte doch etwas sehr Wertvolles sein, etwas Zerbrechliches ... ein Familienschmuck, zum Beispiel, eine alte kostbare Uhr oder auch ... nein, da sollte nichts kaputt gehen. - Das stimmt! ... Jetzt bist aber du daran!

Ich machte also weiter. Zuerst nestelte ich an der Schleife des Stoffbändchens, das die kleine Schachtel zusammenhielt. Dann nahm ich vorsichtig den länglichen Deckel ab. Drinnen, auf dem sandfarbenen Boden der Schachtel, sah ich einen weissen Kartonstreifen, auf dem, ich zählte ... neun, zehn, elf, zwölf, ja, insgesamt zwölf goldene Münzen lagen ... mit einem transparenten Klebestreifen befestigt.
- Tja, Geld hast du von jemandem bekommen, Werner! Zwölf Münzen, vermutlich aus Gold ... schau dir das selber an. Toll!

- Was meinst du? Was bedeutet das?

- Das musst du selber wissen! Einen Gönner hast du da, einen betuchten Sponsor. Schön! Du hast aber auch vielen Leuten geholfen!

Werner löste den Klebestreifen ab, nahm drei Goldmünzen in die Hand und untersuchte sie.

- Alle gleich!

- Zeig mir ein Stück ... ja, relativ schwer. Das kann nur Gold sein.

Er schaute sich das längliche Kartonstück genauer an. Von beiden Seiten. Recht lange.

- Nichts! Ich dachte, vielleicht steht etwas darauf ... ein paar Zeilen, eine Mitteilung.

- Das dachte ich auch! Aber da ist nichts. Ich habe noch einmal in den Plastiksack hineingeguckt. Schau selber!

Werner untersuchte den Sack sehr gründlich und stülpte ihn zuletzt um.

- Leer! Du hast recht!

Er gab mir die zwei übrigen Münzen in die Hand. Ein männlicher Kopf auf der einen, ein Adler auf der anderen Seite. Lateinische Schrift ... zu klein, ohne Brille konnte ich das nicht entziffern. Und dennoch, ein wenig grösser, eine Zahl: 1967.

- Hast du das auch gesehen? Aus dem Jahre siebenundsechzig. Es muss Gold sein, da bin ich mir ziemlich sicher! Sind alle Münzen gleich alt?

Er schaute: - Siebenundsechzig, siebenundsechzig ... da, diese eine! Da steht neunzehnhundertfünfundsechzig. Ist sie deshalb auch etwas wertvoller?

- Nein, nicht unbedingt. Das ist kein antikes Geld. Das Gold selbst macht hier in erster Linie den Wert aus, nicht die Prägung.

- Was könnte ich wohl für alle kriegen? Oder ... für eine Münze?

- Ach Werner, das weiss ich nicht! Ich schätze fünf bis siebenhundert D-Mark pro Stück. Auf der Bank könnte man es dir genauer sagen.

- Was, siebenhundert D-Mark? Huiii!

- Vielleicht etwas weniger - oder auch mehr; wirklich, keine Ahnung!

Werner nickte, dann schüttelte er den Kopf und nickte wieder.

- Das kann nicht wahr sein!

- Es ist wahr, ja, es ist wirklich so! Das ist dein Geld!

- Gold!

- Du sagst es!

Er steckte die zwölf goldenen Münzen in seine Bluejeans und legte, sorgfältig und sehr langsam, das Packpapier zusammen. Dann schob er es in den bunt bedruckten Plastiksack.

- Also!

- Gehen wir? 
VI

Werner hatte es verdient! Wer der edle Spender sein könnte, nein, das bereitete mir kein grosses Kopfzerbrechen. Es handelte sich wahrscheinlich um jemanden vom Dorf - drei oder vier Familien, vermögendere Leute, kamen wohl in Frage - mit Auswärtigen hätte man aber ebenfalls rechnen müssen; Werner war doch als Hilfsgärtner auch ausserhalb unserer Gemeinde $a b$ und zu tätig.

Und er selber? Wie ging er mit dem grossen Fragezeichen um? War er neugierig? Oder ... wollte er überhaupt wissen, wer von seinen Arbeitgebern der grosszügige Sponsor war?

Vielleicht. Ich wusste es nicht. Nach meinen Ideen oder diesbezüglichen Vermutungen fragte er mich nie.

Werner ist also - für seine Verhältnisse - wohlhabend geworden. Aber zufriedener war er deshalb nur in den ersten zwei, drei Wochen.

Nach wie vor ging es ihm gesundheitlich nicht besonders gut. Im Gegenteil! Die Schmerzen unter dem rechten Rippenbogen nahmen zu, nach einer fetten Mahlzeit - eine kalorienreiche Hühnersuppe in erster Linie - musste er sich oft übergeben. Ich brachte ihm zwar aus der Schweiz ein Leberenzym-Präparat mit, er mochte aber die grossen Tabletten nicht schlucken, er liess sie unberührt.

Sein Hauptproblem lag eben anderswo. Es war das Geld, seine zwölf Goldmünzen.

Dass er sie nicht ewig in seiner Hosentasche tragen konnte, das wusste er. Er ahnte sicher auch, dass es unvernünftig gewesen war, im Dorf die radikale Veränderung seiner Besitzverhältnisse bekanntzugeben. Und gerade dies hatte er getan ... im Konsumladen, wo er durch die Erwähnung seines neuen Wohlstandes die Vergrösserung seiner Kreditwürdigkeit erhoffte.

Die Folge wurde ihm nun klar; leider zu spät!

Zu Hause - im Küchentisch, unter dem Kissen oder in einer Schublade im Schrank - könne er das Gold nicht liegen lassen, sagte er; die "Villa" sei praktisch nicht abschliessbar. Und auch wenn er seine spärliche Barschaft in ein Hängeschloss investierte, auch dann wären die Sorgen nicht behoben; das Haus wäre für den Fremden, Unbefugten immer noch nicht unzugänglich gemacht, denn: Das Dach sei ein Problem! Nur ein paar Dachziegel bräuchte der dreiste Dieb wegzunehmen, durch das Loch hineinzusteigen - und schon wäre er in der Wohnstube!

Offensichtlich verfolgten ihn die bösen Gedanken auch in der Nacht. Am Anfang des Sommers beklagte er sich vor allem über hartnäckige Schlafstörungen. Tägliche Rückenschmerzen kamen noch hinzu.

- Werner, sagte ich, - irgendwann musst du dich entscheiden. Das Problem ist sicher lösbar. Klar ist nur eins: Das Geld, das ganze Geld, das du nun hast, nur in deiner Jeanstasche zu tragen, nein, das geht auf die Dauer nicht.

- Lösbar, lösbar ... verrate mir nur wie!

- Es gibt, Hunderte, ja Tausende von Möglichkeiten. Verstecke das Gold zum Beispiel irgendwo!
Schneller gesagt als getan! Das sei doch seine erste Idee gewesen, meinte er, die er zu Beginn gehabt hätte.

Nur langsam liess er sich seine Überlegungen und Bedenken aus der Nase ziehen ... den Grund also, warum er sich zuletzt gegen ein sicheres Versteck entschieden hatte.

Im wesentlichen ging es um folgendes: Er traute seinem eigenen Gedächtnis nicht über den Weg. Er war sich nämlich keineswegs sicher, ob er in der Lage wäre, den versteckten Goldschatz wiederzufinden.

- Helmut, sagte er zum Schluss, Helmut, du bist mein Freund und dank dir habe ich das Geld überhaupt gefunden.

- Mit der Zeit wäre dir der Plastiksack auch in den Sinn gekommen ... da bin ich mir ziemlich sicher!

- Gut, gut! Du könntest mir aber weiterhelfen.

- Wenn das in meiner Macht steht, gerne. Wie?

- Na ja ... vielleicht würdest du die Münzen zu dir nehmen, sie bei dir verstecken. Dein Haus kann man doch ohne Probleme abschliessen!

- Okay, das kann man ... aber ich weiss nicht, Werner, lieber würde ich es nicht tun. Und wenn, wenn überhaupt, dann nur als letzte Möglichkeit.

- Wie du meinst!

- Oh nein, sei bitte nicht gleich eingeschnappt! Das war kein hundertprozentiges "Nein». Wie ich schon sagte: Alles bei mir deponieren ... nur wenn uns nichts Besseres einfällt. Auch wenn das Gold bei mir ist, Werner, tja, auch dann gäbe es noch einen Haken.

- Ja?

- Nun, was wäre, wenn mir zum Beispiel etwas zustossen würde? Dass ich deshalb eine schriftliche Erklärung bei einem Rechtsanwalt hinterlasse, das wünscht du dir vermutlich nicht.

- Richtig!

- Also! Lass uns noch in Ruhe nach anderen Wegen suchen!

Er war einverstanden. Suchen wollte er, er wusste bloss nicht wie und in welcher Richtung. Und ich hielt mich mit meinen Vorschlägen lieber zurück.

\section{VII}

Im Laufe der Sommermonate muss Werner weiter abgenommen haben. Wie schwer er vorher war, wussten weder er noch ich genau, ich schätzte aber, der Unterschied machte fünf bis sechs Kilo aus. Hosen und Pullover hingen an ihm wie an einer Vogelscheuche. - Werner, ich kann nicht zuschauen! sagte ich. Etwas musst du schon unternehmen ... in deinem eigenen Interesse.

- Ich weiss, ich weiss! Die Schmerzen sind aber weg. Und jetzt habe ich ganz andere Sorgen. Alles wird gemacht, wie du es dir wünschst ... nur später.

- Klar, ich verstehe dich, und trotzdem ...

- Helmut, was willst du überhaupt?

- Ich? Ich hoffe, du auch, wir beide. Eine komplette internistische Abklärung. Herz, Nieren, Nebennieren, Magen, Dünn- und Dickdarm ... alles. Und vor allem: die Leber! 
- Ich bin nicht versichert.

- Ich weiss.

- Das kostet viel Geld.

- Eben, mein Lieber, eben! Jetzt wäre gerade die

ideale Zeit für eine Untersuchung! Kohle hast du ja!

Werner wirkte nachdenklich. Er senkte den Kopf und kratzte mit der Schuhspitze an meinem Stuhl. Eine Weile sass er schweigsam, nach vorne gebeugt.

- Nun?

Er hob den Kopf. Und er lächelte.

- Die Idee finde ich gut! Ich weiss dann, woran ich bin. Und vor allem ... das Geld bin ich los! Nicht schlecht!

- Das ganze Geld müsste nicht gleich draufgehen, hoffentlich nicht ... Deine Geldvorräte würden natürlich schrumpfen. Auf die Hälfte schätze ich ... das weiss ich aber nicht so genau. Je nachdem, wo du dich untersuchen lässt. Wenn du in die Schweiz fahren willst ...

- Sollte ich?

- Nein, das wollte ich damit nicht sagen! Das ist deine Entscheidung.

- Was meinst $d u$ aber?

- Ich weiss nicht. Ich kenne mich da zuwenig aus. Ich glaube allerdings, auch hier gibt es gute Ärzte; in Luino, in Laveno, Gallarate ... und vor allem in Varese, das ist eine grössere Stadt. Du musst dich halt richtig informieren lassen!

- Mache ich, das mache ich, Helmut. Das kann ich dir versprechen!

Er tat es, er hielt sein Wort; das erfuhr ich von Signore Alberto Bianchi, dem Leiter des hiesigen Lebensmittelladens.

Ja, ja, viele Menschen habe Werner nach den hiesigen Ârzten gefragt, meinte Herr Bianchi. Ob sie gut oder schlecht seien, feinfühlig oder grob, wollte er wissen; und überhaupt - was er jetzt machen solle, welches Spital wäre zu empfehlen ... gut und nicht sehr teuer. Alles habe geklungen, als ob er schleunigst eingeliefert und unters Messer kommen müsste. Welche Meinungen oder Empfehlungen Werner bekommen hätte ... das wisse er natürlich nicht so genau.

Als wir uns ein paar Tage danach wieder sahen, fragte ich ihn: - Also, was nun, Werner? Wie geht's dir? Und wie steht es mit den Schmerzen?

- Ich habe keine, sagte er. - Seit längerer Zeit gar keine mehr ... Gott sei Dank! Das ist doch wichtig!

- Das schon, Werner, das ist sehr wichtig. Aber das ist nicht alles!

- Die meisten haben gemeint, sich nur so, ohne klare Schmerzen, operieren zu lassen ... das habe keinen Sinn!

- Was? Wieso operieren? Wie kommst du darauf? ... Nur untersuchen lassen, eine gründliche Untersuchung hinter dich bringen, verstehst du? Dann wüssten wir, woran wir sind ... vor allem mit der Leber und mit der heimtückischen Gewichtsabnahme. Mit der ist das nicht so problemlos, mein Lieber. Die hat ihren Grund!
Werner widersprach nicht. Er kratzte nun wieder mit der Schuhspitze an dem Stuhl bei meinem Schreibtisch und meinte, dass die Schmerzen jetzt vorbei seien, das freue ihn am meisten.

- Mich auch, Werner, chronische Schmerzen, ja, das ist etwas Schreckliches, ich weiss.

Dann müsse ich ihn also verstehen. Die anderen Sorgen seien viel wichtiger.

- Mit dem Geld?

- Ja! Ich denke immer wieder daran. Und auch mit dem Schlaf ist es noch um keinen Deut besser. Allerdings ... ich habe jetzt eine neue Idee.

- Prima! Raus damit!

- Ich könnte die Münzen irgendwo vergraben.

- Ach!

- Ja, zuerst gut einpacken ... und in ein Loch damit!

- Merkst du dir dann, wo du deinen Schatz vergraben hast?

- Ich glaube schon. Aber ich verlasse mich nicht darauf.

- Sondern?

- Ich mache mir einen genauen Plan.

- Ja ...

- Einen Situationsplan meines Grundstückes habe ich mir schon im Dorf bei der Gemeindeverwaltung geholt.

- Gut, das wäre der erste Schritt.

- Und der Wichtigste!

- Werner, merkst du dir aber, wo du den Plan versteckt hast? Wenn nicht, wären wir so weit, wie wenn du das Geld irgendwo vergraben würdest.

Werner schwieg, das war für ihn offensichtlich keine einfache Frage. Ob er dann den Plan finden würde? Das wusste er auch nicht.

- Was meinst du?

- Schwer zu sagen, Helmut. Lass mich nachdenken. Ich öffnete lieber keine Flasche Wein ... zumindest bis alles etwas klarer würde. Ich goss uns Schwarztee ein.

- Helmut, kennst du Metalldetektoren?

- Ja, aber nur am Rande.

- Das sind doch Geräte, die Metall aufspüren können. Auch in der Erde. Stimmt's?

- Ja, es stimmt.

- Wenn ich so etwas hätte, dann dürfte das Finden kein Problem sein ... wenn ich den Plan irgendwo verlegen sollte.

- Ja, das sehe ich ein.

- Also! Zuerst müsste ich mir einen solchen Detektor besorgen.

- Billig ist so ein Gerät nicht ... mach dir da nichts vor!

- Hunderttausend Lire? Oder noch mehr?

- Oh, viel mehr, Werner!

- Das kann man schnell abklären!

Ich schwieg eine Weile. Er lächelte verschmitzt und schlürfte an seinem Tee. Keine Beanstandungen wegen des fehlenden Weines. 
- Werner, jetzt ganz im Ernst: Willst du dir wirklich ein solches Gerät anschaffen? So viel Elektronik auf einem Haufen! Du warst immer ein ausgesprochener Technikgegner. Keine elektrische Stichsäge, Schleifmaschine, Benzinsäge ... nichts von alledem durfte je in deine Villa kommen. Nicht einmal einen Schubkarren wolltest du benutzen, um das Notwendige für deinen Zaun zu transportieren! Oder ...

- Lass das, Helmut! Ich weiss, du hast recht.

- Es geht nicht um recht haben, es geht um die Sache selber. Noch einmal also: Was machen wir mit deinem Schatz? Das Ganze plagt dich, man sieht es dir an. Du isst kaum etwas, auch der Schlaf hat sich nicht gebessert ... wenn du meinst, wir sollten es bei mir zu Hause lassen, gut, ich ...

- Nein, nein! Ich finde schon einen Weg ... ich brauche nur ein wenig Zeit.

Er lächelte nachdenklich und hielt mir seine leere Teetasse hin. Ich schenkte ein.

- Ich weiss, sagte er. - Grande problema, molto grande!

\section{VIII}

Die erste Septemberwoche. Nicht nur der Monte Zeda und der Lauraskaberg, auch ein paar nähere Bergspitzen zeigten sich am Vormittag weiss.

Am Wochenende hatte ich Verwandte aus Deutschland bei mir, meinen Cousin und seine Frau; am Montag stieg ich aber schon um neun - frühe Besuche schätzte Werner nicht - hinauf zu seiner "Villa".

Ich klopfte ... nichts. Ich wartete eine Weile, klopfte noch einmal, wieder keine Antwort ... Ein paar Sekunden stand ich vor der Tür und horchte. Stille. Dann trat ich ein.

Werner schlief ... schön eingewickelt in alle Decken, die er besass. Ich überlegte mir kurz, was ich machen sollte. Ihn wecken? Nein, dazu hatte ich keine grosse Lust. Gleich wieder hinuntergehen, ins Dorf? Das gefiel mir auch nicht besonders. Ich stand eine Weile herum und setzte mich dann auf einen Stuhl beim Esstisch.

Der Stuhl knarrte; kein lautes Geräusch, es reichte aber, um Werner zu wecken. Er machte die Augen auf und lächelte.

- Du bist es!

- Ja, ich kam gerade ... ich wollte dir etwas bringen.

- Mir? Was denn?

- Die Ordner ... für deine Zeitungen und Journale.

- Aber keine neuen!

- Nein! Ich weiss, das war dein ausdrücklicher

Wunsch. Second hand!

- Helmut ... schwindelst du jetzt nicht?

- Nein! Warum meinst du?

- Alte, gebrauchte Ordner, die suche ich schon lange. Die gibt es aber nicht! Wenn der Rücken ein paar Schrammen hat, werden sie offensichtlich gleich weggeschmissen ... tja, so sind halt die Menschen jetzt!

- Das mag stimmen. Diese sind aber schon gebraucht, wie du es haben wolltest ... schau!
- Woher hast du sie? Das solltest du mir verraten! - Ah, wenn du es unbedingt wissen willst: Von mir. In der letzten Woche habe ich mir neue gekauft, ein bisschen dicker sind sie, das war mir wichtig. Meine alten Leitz-Ordner sind also überflüssig.

Werner lächelte mich an. - Vielen Dank! In den nächsten Tagen wird alles schön versorgt ... dann sind die Stühle wieder frei. Und jetzt stehe ich auf!

- Nur wenn du magst ... nicht meinetwegen. Ich wollte kurz vorbeischauen, gucken, wie es dir so geht. Vierzehn Tage warst du nicht mehr bei mir!

- Schon so lange?

- Ja, zwei Wochen. Geht's gut?

- Wie immer ... mal besser, mal schlechter.

- Falls du aufstehen willst, könnten wir langsam ins Dorf marschieren. Und etwas Kleines bei mir zu Mittag essen.

- Ja, gerne, vielen Dank! Hoffentlich schaffe ich das. Seit ein paar Tagen fühle ich mich morgens so schwindlig.

- Auch ohne Alkohol, Werner, auch wenn du am Vorabend trocken warst?

- Klar, auch dann! Wein habe ich seit mehr als einer Woche nicht mehr angerührt. Schau dich ruhig um. Keine leere Flasche findest du im Haus ... Du glaubst mir nicht?

- Wenn du es sagst, glaube ich dir. Und das mit dem Schwindelgefühl ... ich weiss nicht, auch das gefällt mir nicht besonders. Irgendeinen Grund dafür muss es doch geben - wir kennen ihn bloss nicht! ... Hast du dich schon beim Arzt angemeldet?

Er schwieg. Die Augen geschlossen. Ich wusste nicht, ob er nicht wieder eingeschlafen war.

- Hörst du mich, fragte ich leise.

- Klar höre ich dich! Du musst nicht gleich flüstern!

- Ich meine ...

- Ich weiss, was du meinst! Was soll ich dir aber sagen? ... Nein, weder im Spital war ich noch bei einem Arzt ... Bist du jetzt zufrieden?

- Du wolltest doch abklären, ob ...

- Hab ich auch gemacht! Ich bleibe in Italien. In einem Schweizer Spital wäre alles viel zu teuer; mit Locarno und Bellinzona habe ich telefoniert ... vor drei Wochen, bald nach unserem letzten Treffen.

- Gut, also hier! Das ist in Ordnung. Du solltest das nur nicht allzu lange vor dir herschieben. Gewartet haben wir schon lange genug.

- Ja, ich mache es!

Er setzte sich auf den Bettrand und zog langsam seine dicken Socken an. Dann stand er auf, hielt sich am Kreuz und stöhnte ein wenig.

- Rücken? Wie bei mir!

Keine Antwort. Er gähnte noch, nahm von einem Kleiderhaken bei der Eingangstür einen wollenen Pullover ab und streifte ihn über.

- Also, Werner, was ist jetzt los? Sprichst du nicht mehr? 
- Doch, doch! Ich weiss nur nicht, was ich dir sagen soll. Im Prinzip hast du in allem recht. Aber was kann ich damit anfangen? Was kann ich überhaupt mit mir anfangen ... muss ich noch hier sein? Warum ... wozu? Du weisst es vielleicht ... für dich. Für mich bin ich mir nicht mehr im klaren.

- Das kenne ich, Werner! So etwas kennen eigentlich fast alle Menschen über fünfzig. Einmal sieht man alles sehr optimistisch gestimmt und dann wieder nur grau und schwarz. Warum solltest du da eine Ausnahme machen?

- Meinst du?

- Aber sicher!

- Ich bin noch nicht fünfzig.

Für einen Augenblick geriet ich ins Stocken. Ich hatte ihn zwar nie nach seinem Alter gefragt, schätzte ihn allerdings knapp unter sechzig.

- Gut! Aber wahrscheinlich bald.

Werner nickte. - In drei Monaten.

Immer noch kein Lächeln, trotzdem wirkte er ein wenig entspannter und zufriedener.

Er zog sich an. Wieder seine Bluejeans - ich glaube, die anderen Hosen, die dünnen, für den Sommer, besass er nicht mehr; zu löcherig waren sie und auch zu weit; sein Po und seine dünnen Beine verschwanden darin.

- Gehen wir, Werner?

- Von mir aus! Ich bin bereit.

\section{IX}

In den folgenden zwei Wochen gab es oft starke Winde, der See erschien von unserem Berg aus hellgrau, fast weiss; meistens hatten wir aber sonniges Wetter. Dann kamen plötzlich - für Natur und Menschen völlig unerwartet - kalte Tage. Schnee lag auf den Nordhängen und reichte tief in die Täler herunter.

Werner machte mir nun Sorgen. Am Wochenende brach ich zu ihm auf. Eine halbe Stunde später stand ich vor seiner Holzhütte; ich klopfte, wartete nur kurz und trat ein.

Es war gut aufgeräumt, für Werners Verhältnisse sogar erstklassig, aber sehr kalt. Ein geleerter Aschenbecher stand auf dem Tisch - ungewöhnlich - und sein niedriges Bett ist schon gemacht.

- Werner, rufe ich, - ich bin's, Helmut!

Nichts! Auch kein Knarren der Balken oder das Summen des laufenden Wasserhahns hinter dem Plastikvorhang.

- Werner, ich bin da!

Keine Antwort. Ich setze mich an den Tisch, halte aber die Stille nur ein paar Sekunden aus; ich verlasse die Hütte. Draussen schaue ich mich überall um, gehe zum Holzschuppen, gucke hinein ... und marschiere um das Haus herum. Werner nirgends.

Ich warte bis zum Mittag. Dann schreibe ich ihm auf einen Zettel, dass er sich bei mir melden soll, und kehre ins Dorf zurück.
Der Sommer ist vorbei, gestern war der erste Oktober. Kalt. Und sehr windig.

Niemand hat Werner gesehen, niemand weiss etwas von ihm. Vorgestern wurde ich von zwei Polizisten ausgefragt. Vorläufig steige ich noch jeden Tag zu seinem Haus hinauf; ich suche ihn im Wald und sitze in seiner Hütte. Langsam schwindet aber meine Hoffnung.

In unserer Dorfkneipe ist Werner seit fast einem Monat das Hauptthema. Viele Möglichkeiten werden genannt, ernst nehmen - oder sogar verfolgen und prüfen - kann ich aber keine.

Einige Tage später. Sonnig, windstill und für die Jahreszeit ungewöhnlich warm. Föhn.

Die Hoffnung, dass ich Werner je lebendig wiedersehen werde, ist relativ klein ... da will ich mir nichts vormachen. Irgendwo muss er aber sein weder hat er sich in Luft aufgelöst, noch ist er in der Erde verschwunden - nur wo? Liegt er im See? Oder auf dem moosigen Boden im Wald? Weit von seiner Hütte? Oder ziemlich nah?

Ich suchte ihn. Immer, wenn es das Wetter erlaubte. Auch gestern war ich unterwegs. Mein Ziel der Monte Casso.

Er liebte doch diesen Berg! Dort war er oft hingegangen und immer heiter und zufrieden zurückgekommen. Er wunderte sich nur, dass niemand von uns Neuen im Dorf dieses wunderbare Massiv kenne und bereit sei, mit ihm dort hinaufzusteigen. Gut, ein wenig unbequem sei der Weg schon, sagte er; zu Beginn sehr heiss, Südseite, und später ziemlich steil. Insgesamt zweieinhalb bis drei Stunden ... wenn man ein wenig trainiert sei und flott gehen wolle. Aber es lohne sich! Der Ausblick von oben sei einmalig! Er habe nirgends sonst so etwas Wunderbares gesehen.

Der Weg war bezeichnet, etwa bis zur Hälfte. Dann hörten die weiss-gelb-weissen Markierungen auf; von dort war es aber nicht mehr schwierig, die Richtung zu halten - man musste bergauf, immer weiter hinaufgehen.

Ein einzigartiger Wald! Tannen gab es selten, nur kleine Gewächse; Buchen und Esskastanien vorherrschend, auch Birken standen vereinzelt da. Kaum ein Stamm war aber breiter als zehn Zentimeter. Und dazwischen alte, siechende Kastanienbäume; oft hohl, morsch, ein paar Seitenäste ragten aus dem kranken Rumpf, der in zwei, drei Metern Höhe plötzlich aufhörte. Unten aber, in Bodennähe, musste der Baumstamm zumindest einen Meter breit sein.

Je höher ich kam, desto kleiner waren die jungen Buchen und Birken, um so häufiger begegnete ich allerdings den verstümmelten Riesen. Als ich an ihnen die ersten verkohlten Stellen bemerkte, wurde mir klar: Hier hatte es gebrannt! Es liegt schon Jahre, ja, vielleicht Jahrzehnte zurück, es muss aber ein fürchterlicher Waldbrand gewesen sein. 
Weiter! Der Boden wurde immer steiniger. Kleine, dunkelbraune Eidechsen huschten vor mir weg. Die verstümmelten Baumriesen hörten auf, die Birken und Buchen wurden noch dünner und mickriger; die letzten drei, vierhundert Meter vor dem Gipfel gab es auch die nicht mehr.

Der Wind blies nun etwas stärker, ein paar Wolken hüllten den Gipfel ein. Eigenartigerweise sah ich auf einem Felsenvorsprung wieder eine weiss-gelbweisse Markierung. Das beruhigte mich; ich befand mich also auf Werners Pfad. Ich hielt immer noch Ausschau nach ihm ... und wenn ich ehrlich bin, auch nach Wildschweinen. Die soll es hier tatsächlich geben, man sagt es im Dorf. Ich überlegte mir, wie wahrscheinlich es wäre, dass mir ein Wolf begegnete, schloss diese Möglichkeit aber aus.

Tja, ich hatte Angst, das will ich nicht leugnen; die empfinde ich eigentlich immer in den Bergen, vielleicht ist das der wahre Grund, warum ich Werner nie Gesellschaft bei seinen Wanderungen geleistet habe.

Ich bedauerte nun, dass ich meinen Entschluss, auf den Monte Casso zu gehen, erst zu Mittag gefasst hatte und deshalb so spät - zu spät - aufgebrochen war.

Drei Uhr. Keine Wetterverschlechterung, Gott sei Dank! Nur die Windstärke nahm weiter zu, ich empfand sie aber nicht als beunruhigend. Im Gegenteil! Die Wolken wurden nun vollständig weggepustet, der Gipfel schien plötzlich nah zu sein. Schien es nicht nur ... er war es auch! Kaum eine viertel Stunde später stand ich auf dem höchsten Punkt des Berges. Steine, trockenes Gras, grauer Sand.
Erst jetzt setzte ich mich auf den kühlen Boden und drehte mich um.

Werner hatte recht. Die Aussicht war überwältigend!

Die hinteren Bergspitzen schimmerten hellblau, fast weiss. Den Monte Rosa und den Monte Dogano erkannte ich sofort, auch mit dem Broman hatte ich keine Schwierigkeiten. Bei den Gipfeln, die rechter Hand lagen, nördlich also, in Richtung Schweiz, war ich mir nicht mehr sicher. Ich schwor mir, in den nächsten Tagen noch einmal hierher zu kommen mit einem Fotoapparat und einer Wanderkarte.

Vor den entferntesten Bergketten schien ein weisser Schleier zu hängen, sie wirkten leicht, fast durchsichtig. Die näheren Berge waren ein wenig dunkler - ich zählte acht Reihen, acht blau-graue Abstufungen. Nein, Blautöne nahm ich bei den allernächsten Berghängen nicht mehr wahr. Die Wälder oberhalb von Cannobio und Cannero waren grün, hellgrün; diese beiden Ortschaften konnte ich nicht sehen, sie lagen zu tief, zu nah am See, ich erkannte aber die etwas höher gelegenen Dörfer - Bian Cavallo, Novagrio und Preneno.

Ich stützte mich auf die Ellbogen hinter meinem Rücken, ich lag schon fast. Dann kniff ich die Augen ein wenig zusammen und betrachtete die in der Ferne sich langsam auflösenden Bergspitzen und Berggrate.

Du hast recht, Werner, einmalig! Auch ich, das solltest du jetzt wissen, auch ich habe so etwas in meinem Leben noch nie gesehen.

Hell. Alles so hell! 